\title{
PRL3-zumab, a first-in-class humanized antibody for cancer therapy
}

\author{
Min Thura, ${ }^{1}$ Abdul Qader Omer Al-Aidaroos, ${ }^{1}$ Wei Peng Yong, ${ }^{2,3}$ Koji Kono, ${ }^{3,4}$ Abhishek Gupta, ${ }^{1}$ \\ You Bin Lin, ${ }^{1}$ Kousaku Mimura, ${ }^{3}$ Jean Paul Thiery, ${ }^{1,3}$ Boon Cher Goh, ${ }^{2,3}$ Patrick Tan, ${ }^{5}$ Ross Soo, ${ }^{2,3}$ \\ Cheng William Hong, ${ }^{6}$ Lingzhi Wang, ${ }^{3}$ Suling Joyce Lin, ${ }^{5}$ Elya Chen, ${ }^{4}$ Sun Young Rha, ${ }^{7}$ \\ Hyun Cheol Chung, ${ }^{7}$ Jie Li, ${ }^{1}$ Sayantani Nandi, ${ }^{1}$ Hiu Fung Yuen, ${ }^{1}$ Shu-Dong Zhang, ${ }^{8}$ Yeoh Khay Guan, ${ }^{9}$ \\ Jimmy So, ${ }^{9,10}$ and Qi Zeng ${ }^{1}$ \\ IInstitute of Molecular and Cell Biology, Agency for Science, Technology and Research (A*STAR), Singapore. \\ 2Department of Haematology-Oncology, National University Cancer Institute, Singapore. ${ }^{3}$ Cancer Science Institute of \\ Singapore, National University of Singapore, Singapore. ${ }^{4}$ Division of General Surgery (Upper Gastrointestinal Surgery), \\ National University Hospital, Singapore. ${ }^{5}$ Cenome Institute of Singapore, A*STAR, Singapore. ${ }^{6}$ Cleveland Clinic Lerner \\ College of Medicine, Cleveland, Ohio, USA. 'Department of Internal Medicine, Yonsei Cancer Research Institute, \\ Yonsei Cancer Center, Yonsei University College of Medicine, Seoul, South Korea. ${ }^{8}$ Northern Ireland Centre for Stratified \\ Medicine, Ulster University, C-TRIC, Londonderry, United Kingdom. ${ }^{9}$ Yong Loo Lin School of Medicine, National University \\ of Singapore, Singapore. ${ }^{10}$ Division of Surgical Oncology (Upper Gastrointestinal Surgery), National University Cancer \\ Institute, Singapore.
}

Novel, tumor-specific drugs are urgently needed for a breakthrough in cancer therapy. Herein, we generated a first-in-class humanized antibody (PRL3-zumab) against PRL-3, an intracellular tumor-associated phosphatase upregulated in multiple human cancers, for unconventional cancer immunotherapies. We focused on gastric cancer (GC), wherein elevated PRL-3 mRNA levels significantly correlated with shortened overall survival of GC patients. PRL-3 protein was overexpressed in $\mathbf{8 5 \%}$ of fresh-frozen clinical gastric tumor samples examined but not in patientmatched normal gastric tissues. Using human GC cell lines, we demonstrated that PRL3-zumab specifically blocked PRL-3+, but not PRL-3', orthotopic gastric tumors. In this setting, PRL3-zumab had better therapeutic efficacy as a monotherapy, rather than simultaneous combination with 5-fluorouracil or 5-fluorouracil alone. PRL3-zumab could also prevent PRL-3+ tumor recurrence. Mechanistically, we found that intracellular PRL-3 antigens could be externalized to become "extracellular oncotargets" that serve as bait for PRL3-zumab binding to potentially bridge and recruit immunocytes into tumor microenvironments for killing effects on cancer cells. In summary, our results document a comprehensive cancer therapeutic approach to specific antibody-targeted therapy against the PRL-3 oncotarget as a case study for developing antibodies against other intracellular targets in drug discovery.

Authorship note: M. Thura and A.Q.0. Al-Aidaroos contributed equally to this work.

Conflict of interest: Q. Zeng is the founder of Intra-ImmuSC, a spin-off company granted licensing rights to the intellectual property portfolio behind PRL3-zumab.

Submitted: March 17, 2016 Accepted: May 12, 2016 Published: June 16, 2016

Reference information: JCI Insight. 2016;1(9):e87607. doi:10.1172/ji.insight.87607.

\section{Introduction}

Although monoclonal antibodies have demonstrated remarkable efficacy as cancer therapeutics (1), only a limited number of extracellular oncotargets have been explored to date, primarily because only extracellular antigens have been regarded as accessible to antibody therapy. As a consequence, intracellular antigens, which have been considered "undruggable" antibody targets, have been traditionally inhibited with small molecules, despite the poorer target selectivity and, consequently, off-target side effects and clinical failures associated with this method $(2,3)$.

To address this gap, in 2008, we proposed an unconventional approach by selecting oncotargets from the large pool of unexplored, previously deemed "undruggable," intracellular oncoproteins for antibody therapy (4). We demonstrated that specific antibody-antigen recognition was essential and sufficient for efficient suppression of tumors expressing the intracellular oncotargets in vivo. Subsequently, we followed up on these findings with a proof-of-concept study validating the feasibility and efficacy of this concept by targeting additional endogenous and exogenous intracellular "tumor-specific antigens" with antibody therapy and vaccination in wild-type C57BL/6 and transgenic spontaneous breast tumor MMTV-PyMT mice 
(5). Since then, three possible mechanisms for the antitumor activity of such intracellular tumor antigenspecific antibodies have been proposed, including antibody penetration into cells, antibody binding to externalized antigen, and/or antibody recognition of MHC-bound antigen-derived peptides $(6,7)$. Collectively, this cancer immunotherapy approach demonstrates that intracellular oncoantigens are indeed tractable to antibody therapy in vivo, creating potentially new growth avenues for the future of antibody-based cancer therapy $(1,8)$.

The most promising intracellular oncotarget from our early studies was phosphatase of regenerating liver 3 (PRL-3, also known as PTP4A3), a member of the PRL family of dual-specificity protein tyrosine phosphatases that we identified in 1998 (9). PRL-3 is localized to the cytoplasmic face of the plasma membrane and endosomes via its prenylated C-termini (10). In 2001, Vogelstein and colleagues first characterized PRL-3 as a metastasis-associated phosphatase, with specific upregulation in metastatic colorectal cancer samples but not primary cancers and normal colorectal epithelia (11). Elevated PRL-3 expression was also independently identified as the most significant predictor of metastatic recurrence in uveal melanoma patients (12). Mounting evidence suggests that PRL-3 promotes multiple stages of malignant transformation (13) via activation of PI3K/Akt, ERK, and SRC oncogenic pathways through downregulation of PTEN (14) and/or activation of upstream receptor tyrosine kinases (15-17). To date, elevated $P R L-3$ mRNA expression levels have clinically been shown to correlate with higher metastatic potential and poor prognosis of multiple cancer types (18).

Gastric cancer (GC) ranks as the third leading cause of cancer mortality worldwide, with more than 700,000 GC-related deaths annually (19), largely due to delayed detection and the asymptomatic nature of the disease in its early stages, coupled to the high rate of recurrence after treatment (20). PRL-3 was first linked with GC progression in 2004 when it was found that higher PRL-3 levels correlated with increased GC invasiveness and metastasis (21). Since then, PRL-3 has been reported to be overexpressed in up to $70 \%$ of primary gastric carcinomas, with higher PRL-3 expression correlating with shorter postoperative survival at all tumor stages in GC patients $(22,23)$, underlining the particularly valuable prognostic potential of PRL-3 in this morbid disease. Radical surgery is the first line treatment for GC, with adjuvant chemotherapy often given before and/or after resection $(24,25)$. However, overall survival with chemotherapy remains poor and is accompanied with undesirable side effects due to nonspecific targeting of other actively dividing, noncancerous cells (20). To this end, targeted therapy using tumor-specific biological agents, particularly antibodies, has emerged as the frontier of anticancer drug development due to their selective inhibition of specific molecules involved in the growth and survival of cancer cells, while leaving normal cells unharmed. In GC, for example, the HER2/neu receptor antagonist trastuzumab (Herceptin) has been approved to target the $13 \%-20 \%$ of GCs expressing cell surface HER2/neu receptors, particularly metastatic gastric or gastroesophageal junction adenocarcinoma (26, 27). However, despite moderate responses, patients often develop resistance to trastuzumab (28), hindering its efficacy. Hence, better tumor-specific molecular targets and/or alternative therapeutic strategies for GC are desperately needed.

In this study, we explored the potential value of PRL-3 as a tumor-specific oncotarget in gastric clinical samples as well as its suitability for antibody therapy to suppress PRL- $3^{+}$gastric tumor growth. Following the success of murine and, more recently, chimeric (29) anti-PRL-3 antibodies in blocking PRL-3-expressing tumors, we herein advance this concept in four key aspects: (a) the use of PRL-3-humanized antibodies (PRL3-zumab), instead of mouse or chimeric antibodies to target PRL-3+ tumors; (b) the establishment of more clinically relevant orthotopic gastric tumor models, instead of experimental mouse tail vein metastasis models; (c) the characterization of PRL3-zumab's superior efficacy as a monotherapy, rather than simultaneous combination therapy with 5-fluorouracil (5-FU); and (d) the demonstration of the profound value of PRL3-zumab in preventing cancer recurrence after surgical removal of PRL- $3^{+}$tumors.

\section{Results}

$P R L-3$ is an oncotarget specifically enriched in tumors but not in normal tissues. A pertinent challenge in the development of anticancer-targeted therapy is the identification of tumor-enriched antigens that are preferentially expressed in tumors but not in normal tissues, so as to avoid undesirable off-target effects. We first screened murine normal tissues by Western blotting for endogenous PRL-3 expression. In our full, uncropped blots, PRL-3 monoclonal antibody detects only a single approximately $20-\mathrm{kDa}$ endogenous protein — corresponding to PRL-3's predicted molecular weight — showing that anti-PRL-3 antibody did 
A

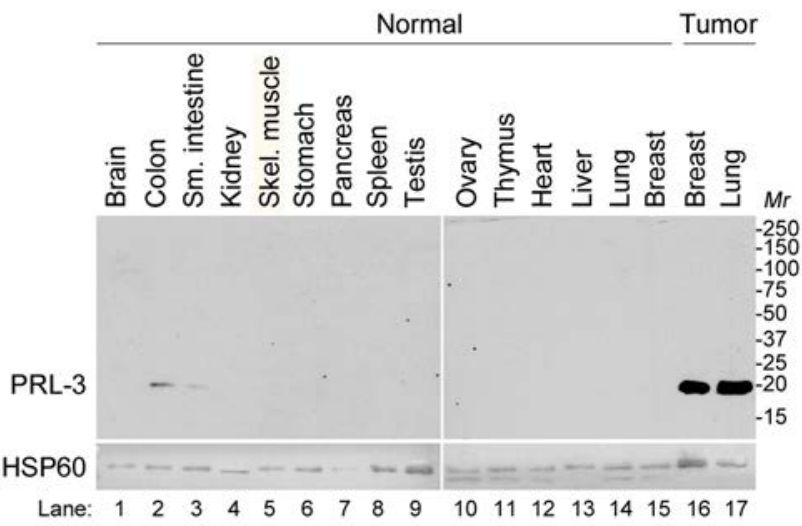

B

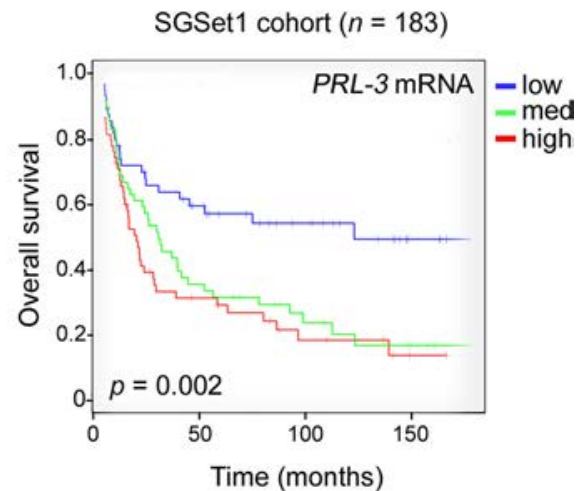

C

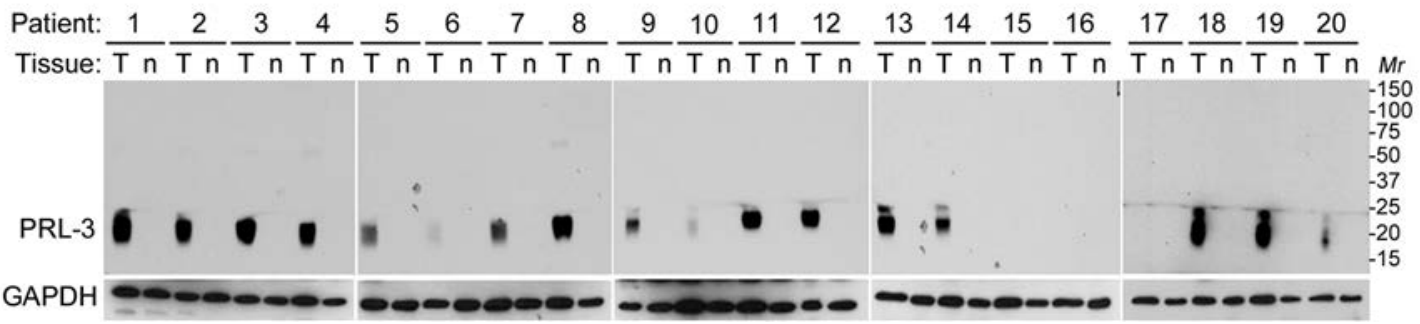

Figure 1. PRL-3 is an oncotarget highly expressed in tumor tissues. (A) Western blots of PRL-3 in various normal tissues of FVB/wild-type mice (lanes 1-15) and spontaneous breast and metastatic lung tumors from FVB/MMTV-PyMT mice (lanes 16 and 17). HSP60, loading control. (B) Kaplan-Meier survival analysis of $P R L-3$ mRNA expression in the Singapore (SGSet1) gastric cancer (GC) patient cohort. $n=183 ; P=0.002$, log-rank test. (C) Full Western blot analysis of PRL-3 in 20 pairs of human primary gastric tumors (T) versus patient-matched normal stomach tissues ( $n$ ) from 20 random GC patients. $\mathrm{GAPDH}$, loading control; $\mathrm{Mr}$, relative molecular mass ( $\mathrm{kDa}$ ).

not cross-react with other molecules (Figure 1A). Although PRL-3 was weakly detected in normal colon and small intestine (Figure 1A, lanes 2 and 3), it was undetectable in 13 other major organs examined (Figure 1A, lanes 1 and 4-15), including normal breast and lung (Figure 1A, lanes 14 and 15). In contrast, PRL-3 was abundantly expressed in spontaneously developed breast and lung tumors (Figure 1A, lanes 16 and 17) from MMTV-PyMT mice (30). In human tissues, PRL-3 was undetectable in most major normal organs examined by immunohistochemistry, with very weak expression detectable in normal colon and small intestine (Supplemental Figure 1A; supplemental material available online with this article; doi:10.1172/jci.insight.87607DS1), mirroring our observation in murine tissues (Figure 1A). Consistent with its tumor-specific upregulation, PRL-3 was undetectable in normal gastric tissue, yet highly expressed in adjacent gastric tumor sections from the same patient (Supplemental Figure 1B). It is worth noting that mice deficient for PRL-3 appear grossly normal (31), suggestive of the dispensability of PRL-3 function in normal tissues. Taken together with our results here, the high expression of PRL-3 in tumor, but not normal, tissues, coupled with the high frequency of PRL-3 overexpression observed in multiple cancers (32), establishes PRL-3 as an ideal tumor-enriched oncotarget.

PRL-3 oncoprotein is overexpressed in $85 \%$ of gastric tumors examined. Over the past decade, a number of studies have demonstrated elevated PRL-3 expression as a negative prognostic factor for GC $(21,33,34)$. We further validated the clinical significance of $P R L-3$ overexpression in GC by two independent approaches. First, we analyzed the clinical characteristics of patients with upregulated $P R L-3 \mathrm{mRNA}$ levels in a cohort of 185 GC patients (clinical characteristics given in Supplemental Table 1). In multivariate Cox analysis, high $P R L$-3 mRNA expression was significantly associated with higher tumor grades (Supplemental Table 2) and shorter overall survival (Kaplan-Meier survival analysis, $P=0.002$; Figure 1B), consistent with independent observations in other GC cohorts $(21,33,34)$. Second, we examined the levels of PRL-3 protein using matched, fresh-frozen biopsy gastric tissue sample pairs (tumor-adjacent normal samples) from 20 randomly selected GC patients. Western blots clearly showed endogenous PRL-3 overexpressed in 17 of 20 (85\%) gastric tumors (Figure 1C). In contrast, PRL-3 was not detected in any matched normal gastric samples 
A

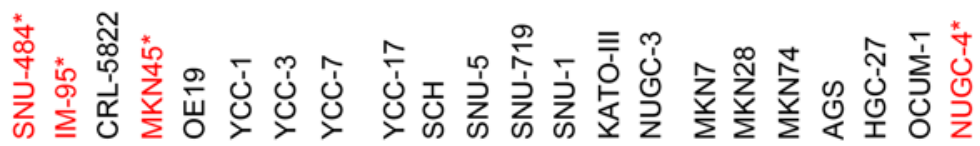

PRL-3

$(\sim 20 \mathrm{kDa})$

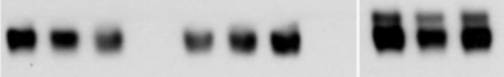

HSP70 - - - - - - - - - - - - - - - - - 70

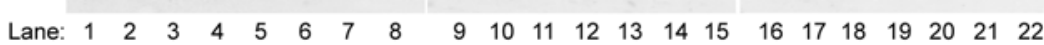

B

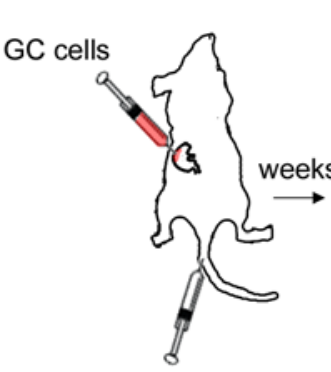

Placebo or PRL3-zumab

(2x/week, i.v.)

D

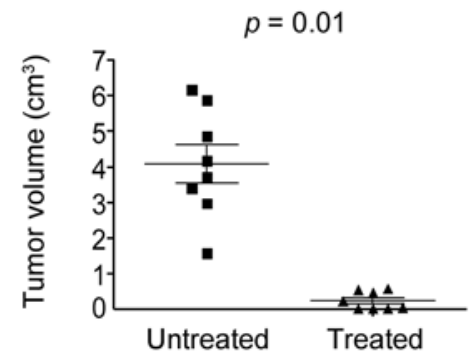

C SNU-484 tumors (PRL-3 ${ }^{+}$)

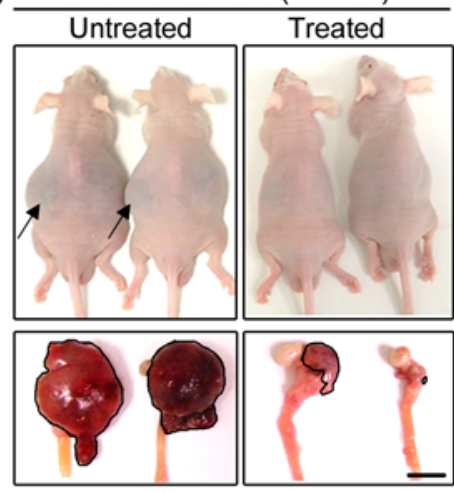

E

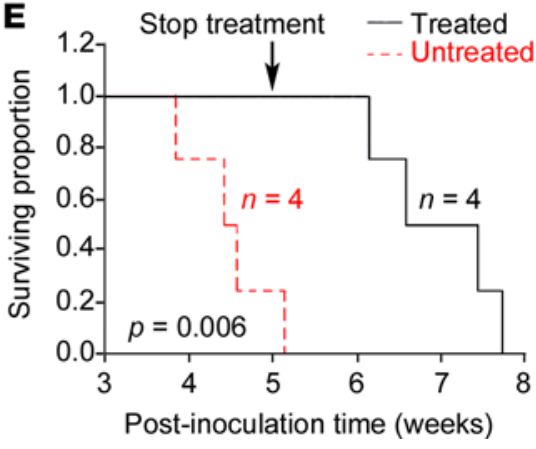

Figure 2. PRL3-zumab specifically blocks PRL-

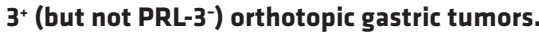
(A) Western blot for endogenous PRL-3 in 22 human gastric cancer (GC) cell lines. Tumorigenic PRL-3+ or PRL-3- cell lines selected for subsequent animal models are indicated with a red asterisk. $\mathrm{Mr}$, relative molecular mass ( $\mathrm{kDa}$ ). (B) Outline of the experimental orthotopic GC model in BALB/c nude mice. (C) PRL3-zumab inhibits PRL-3+ SNU-484 orthotopic gastric tumor growth. Top panels: mouse appearance at the end of the experiment (day 28). Arrows indicate abdominal distention in untreated mice. Bottom panels: excised stomachs with tumor areas framed in black lines. Scale bar: $10 \mathrm{~mm}$. (D) Mean gastric tumor volumes in untreated and treated groups at day 28. $n=8$ per group; $P=0.01, t$ test; data represent mean \pm SEM. (E) Kaplan-Meier survival analysis of untreated (red lines) and treated (black lines) groups of mice. $n=4$ per group; $P=0.006$, log-rank test.

(Figure 1C), validating the tumor-specific expression of PRL-3. Notably, PRL-3 protein appeared as a broad band between 20 and $25 \mathrm{kDa}$ in these full blots, suggestive of extensive potential posttranslational modifications of PRL-3 - such as its previously reported acetylation and/or phosphorylation $(35,36)$ - in human tumor samples. Taken together, the frequent overexpression of PRL-3 in clinical GC samples, coupled with the correlation of elevated $P R L-3$ expression with disease severity, reaffirms PRL-3 as an excellent candidate for targeted GC therapy.

Generation of a PRL-3-targeting humanized antibody, PRL3-zumab. We previously demonstrated the high efficacy of murine and chimeric anti-PRL-3 monoclonal antibodies against metastatic tumors expressing intracellular PRL-3 in both nude and wild-type C57BL/6 mice $(5,29)$. In these studies, mice receiving PRL-3 monoclonal antibodies gained weight continuously and displayed normal activities, suggesting minimal off-target side effects. To translate these early findings towards clinical application in humans, we engineered a humanized monoclonal anti-PRL-3 antibody, hereafter called "PRL3-zumab," based on these scaffolds. Like its predecessors, PRL3-zumab specifically recognizes both mouse and human PRL-3 isoforms equally well and does not cross-react with the homologous PRL-1 or PRL-2 proteins by Western blotting, ELISA, or immunofluorescence analysis (Supplemental Figure 2, A-D). Subsequently, we used PRL3-zumab for all further experiments described in this report.

PRL3-zumab specifically inhibits PRL-3+ but not PRL-3- orthotopic gastric tumors. Human cancer cells growing in their natural (orthotopic) locations in mouse tumor models replicate human disease with high fidelity. More importantly, tumor responses to therapy have been shown to vary dramatically depending on whether cancer cells are implanted in a subcutaneous versus an orthotopic location (37), highlighting the requirement of choosing the right model for assaying therapeutic efficacies of antitumor agents. To establish a relevant preclinical orthotopic murine model to examine the efficacy of PRL3-zumab against gastric tumors, we first screened a panel of 22 human GC cell lines for PRL-3 protein expression status and subsequently tested their tumorigenic capacity within the subserosa layer of stomachs in mice. PRL-3 protein was detected in 13 of $22(59 \%)$ of human GC cell lines analyzed (Figure 2A). However, only a subset 
A

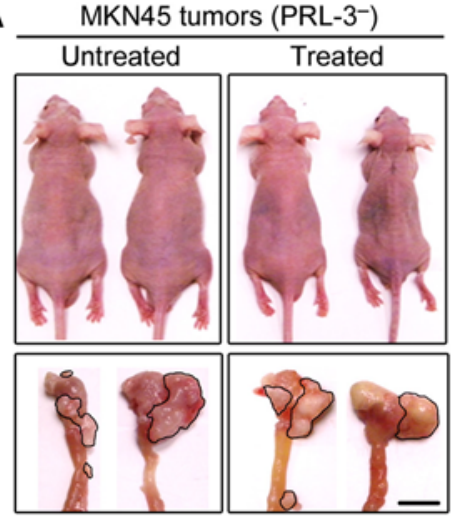

D

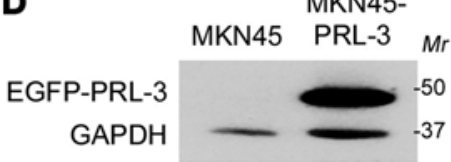

E

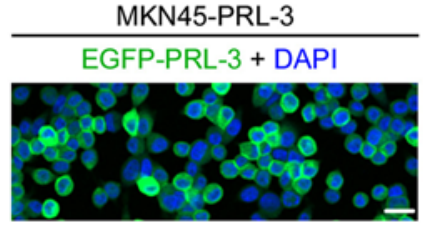

B

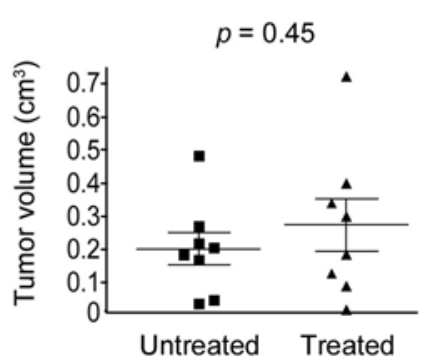

Untreated Treated

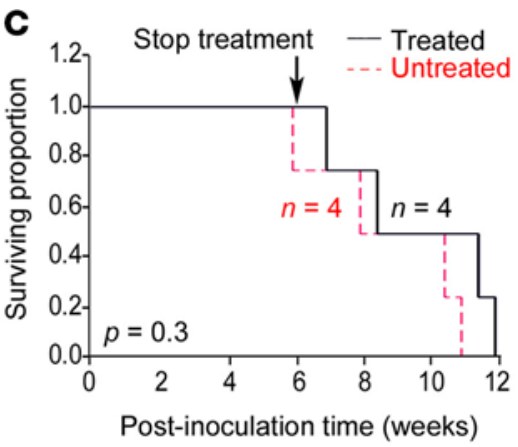

G
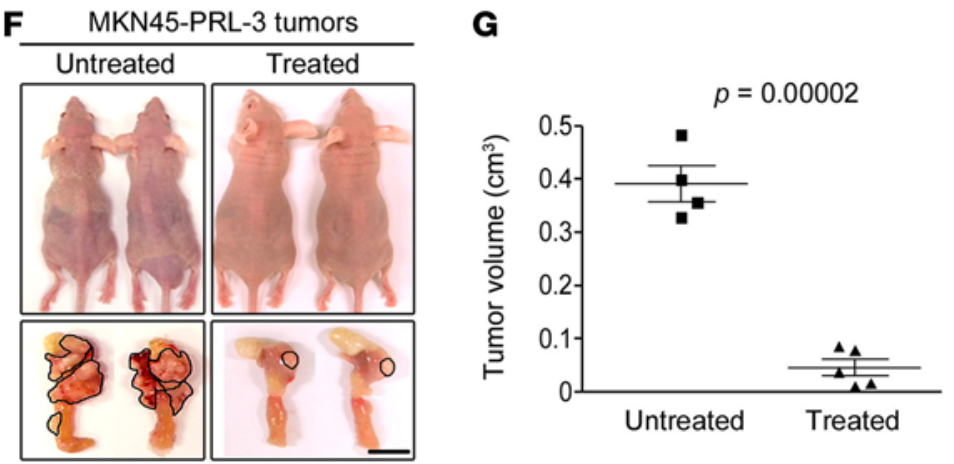

Figure 3. PRL3-zumab has therapeutic effects when negative tumors forced PRL-3 overexpression. (A) PRL3-zumab could not block PRL-3- MKN45 orthotopic gastric tumor growth. Top panels: mouse appearance at the end of the experiment (day 56). Bottom panels: excised stomachs with tumor areas framed in black lines. Scale bar: $10 \mathrm{~mm}$. (B) Mean gastric tumor volumes from $\mathbf{A}$ at day $56 . n=8$ per group; $P=0.45, t$ test; data represent mean \pm SEM. (C) Kaplan-Meier survival analysis of untreated (red lines) and treated (black lines) groups of mice. $n=4$ per group; $P=0.3$, log-rank test. (D) Western blot for overexpressed EGFP-PRL-3 in MKN45-PRL-3 cells. GAPDH, loading control; Mr, relative molecular mass (kDa). (E) Immunofluorescence of the MKN45 cell pool stably overexpressing GFP-PRL-3 (MNK45-PRL-3). Scale bar: $20 \mu \mathrm{m}$. (F) PRL3-zumab effectively blocks MKN45-PRL-3 orthotopic gastric tumor growth. Top panels: mouse appearance at the end of the experiment (day 28). Bottom panels: excised stomachs with tumor areas framed in black lines. Scale bar: 10 $\mathrm{mm}$. (G) Mean gastric tumor volumes from $\mathbf{F}$ at day 28. $n=4$ (untreated group) or 5 (treated group); $P=0.00002, t$ test; data represent mean \pm SEM.

of these GC cell lines grew well in culture and robustly formed orthotopic tumors within a reasonable timeframe ( $<3$ months). Based on these criteria, three PRL- $3^{+}$cell lines (SNU-484, NUGC-4, and IM-95) and one PRL-3- cell line (MKN45) were selected for developing orthotopic GC models to assess the therapeutic efficacy of PRL3-zumab. Cells were inoculated into the subserosa layer of the stomach, and mice were subsequently administered with placebo (untreated) as control or PRL3-zumab (treated) following the protocol outlined in Figure 2B. At the end of the experiment, stomachs were harvested and analyzed for gastric tumor burden.

We first studied the effect of PRL3-zumab treatment on the orthotopic gastric tumors formed by the PRL-3 $3^{+}$SNU-484 GC cell line, which served as an ideal cell line for this study due to its high expression of PRL-3 protein (Figure 2A, lane 1), rapid growth in cultures, and reproducible orthotopic gastric tumor formation within 3 to 4 weeks. Over the course of the experiment (Figure 2B), untreated mice developed pronounced abdominal distention, whereas treated mice maintained a grossly normal appearance (Figure $2 \mathrm{C}$, top). Upon dissection, orthotopic tumor formation was visibly reduced in the treated group compared with the untreated group (Figure 2C, bottom). Measurement of tumor volume revealed a significant 20-fold reduction of tumor burden in the treated group versus untreated group (Figure 2D, $0.23 \pm 0.25 \mathrm{~cm}^{3}$ vs. 4.08 $\pm 1.52 \mathrm{~cm}^{3} ; P=0.01$ ). This phenomenon was unrelated to nonspecific effects of humanized IgG in mice, as administration of human IgG isotype control antibodies did not result in reduced tumor burden (Supplemental Figure 3). Furthermore, despite stopping treatment after 10 doses (5 weeks), treated mice had a significantly longer overall survival compared with untreated mice, with a median survival time of 7 weeks versus 4.5 weeks, respectively (Figure 2E; Kaplan-Meier survival analysis, $P=0.006$ ). These results suggested that mice carrying PRL- $3^{+}$SNU-484 gastric tumors responded effectively to PRL3-zumab therapy. To 
Table 1. Summary of PRL3-zumab treatment outcomes in orthotopic models of $\mathbf{5}$ human GC cell lines

\begin{tabular}{|c|c|c|c|c|c|c|}
\hline \multirow[b]{2}{*}{ Cell line } & \multirow[b]{2}{*}{ PRL-3 status } & \multicolumn{5}{|c|}{ Mean tumor volume \pm SEM $\left(\mathrm{cm}^{3}\right)$} \\
\hline & & Untreated & $n$ & Treated & $n$ & $P$ value \\
\hline SNU-484 & PRL-3+ & $4.08 \pm 0.54$ & 8 & $0.23 \pm 0.89$ & 8 & 0.01 \\
\hline NUGC-4 & PRL-3+ & $0.30 \pm 0.1$ & 4 & $0.02 \pm 0.01$ & 4 & 0.03 \\
\hline IM-95 & PRL-3+ & $0.14 \pm 0.03$ & 6 & $0.01 \pm 0.003$ & 6 & 0.0008 \\
\hline MKN45 & $\mathrm{PRL}_{-3} 3^{-}$ & $0.19 \pm 0.05$ & 8 & $0.26 \pm 0.08$ & 8 & 0.45 \\
\hline MKN45-PRL-3 & $\mathrm{PRL}-3^{+}$ & $0.39 \pm 0.03$ & 4 & $0.07 \pm 0.02$ & 5 & 0.00002 \\
\hline
\end{tabular}

validate this finding, orthotopic GC murine models were generated using two additional PRL- $3^{+}$human GC cell lines, IM-95 and NUGC-4 (Figure 2A, lanes 2 and 22, respectively). Similar to SNU-484 orthotropic tumors, PRL3-zumab treatment resulted in significantly reduced volumes of both PRL-3 ${ }^{+}$IM-95 (Supplemental Figure 4A; $P=0.008$ ) and PRL-3 ${ }^{+}$NUGC-4 (Supplemental Figure 4B; $P=0.03$ ) tumors.

In contrast, gastric tumors formed by the PRL-3- MKN45 GC cell line (Figure 2A, lane 4) showed no response to PRL3-zumab treatment, with pronounced abdominal distention (Figure 3A, top) and orthotopic tumor formation (Figure 3A, bottom). No difference in mean orthotopic tumor volume was found between treated $\left(0.26 \pm 0.22 \mathrm{~cm}^{3}\right)$ and untreated $\left(0.19 \pm 0.13 \mathrm{~cm}^{3}\right)$ groups (Figure 3B; $P=0.45$ ). Likewise, there was no significant difference in overall survival between the two groups, with median survival at 9.25 weeks in the untreated group versus 10 weeks in treated group (Figure 3C; Kaplan-Meier survival analysis, $P=0.3$ ), suggesting that MKN45 tumors lacking PRL-3 oncotarget did not respond to PRL3-zumab treatment. To address the possibility that MKN45 cells might be generally resistant to anticancer therapy, we overexpressed EGFP-tagged PRL-3 in MKN45 cells (MKN45-PRL-3; Figure 3, D and E) and attempted treatment with PRL3-zumab again. In line with PRL-3's pro-oncogenic functions in GC cells, MKN45PRL-3 cells formed aggressive orthotopic tumors in untreated mice (Figure 3F, left) in a shorter time frame than MKN45 cells. Importantly, these pathological phenotypes could be strongly suppressed upon PRL3-zumab treatment (Figure 3F, right). Measurement of tumor volume revealed a significant 10-fold reduction of tumor burden in the treated group versus the untreated group (Figure $3 \mathrm{G}, 0.04 \pm 0.03 \mathrm{~cm}^{3} \mathrm{vs}$. $0.39 \pm 0.06 \mathrm{~cm}^{3} ; P=0.0002$ ). This is in stark comparison with PRL-3- MKN45 parental cells (Figure 3 , A-C), suggesting that while forced PRL-3 expression promoted more aggressive GC tumor growth in vivo, it concurrently endowed responsiveness to PRL3-zumab treatment. Collectively, the results of PRL3-zum$\mathrm{ab}$ treatment of orthotopic tumors derived from these 5 cell lines (summarized in Table 1) cement a fundamental principle underlying PRL-3 antibody therapy (5) - only PRL-3-expressing tumors respond to PRL3-zumab therapy, while tumors lacking PRL-3 expression do not.

PRL3-zumab is more effective as a monotherapy than when coadministered with 5-FU or 5-FU alone. 5-FU is a chemotherapeutic drug used as first-line treatment of GC (20). To study whether PRL3-zumab may synergize with 5-FU in inhibiting orthotopic tumor growth, we tested 4 treatment protocols: placebo as untreated control (group 1), PRL3-zumab monotherapy (group 2), PRL3-zumab and 5-FU combination therapy (group 3), or 5-FU monotherapy (group 4). Dependent on the treatment protocol, biweekly doses of PRL3-zumab (100 $\mu \mathrm{g} /$ dose) or 5-FU (30 mg/kg/dose) were administered individually or in combination i.v. into groups of nude mice carrying orthotopic PRL-3+ SNU-484 gastric tumors. PRL3-zumab monotherapy (group 2) showed the highest therapeutic efficacy, with the lowest mean tumor volume of $0.67 \pm 0.59 \mathrm{~cm}^{3}$, followed by the PRL3-zumab and 5-FU combination treatment (group 3; $1.49 \pm 0.27 \mathrm{~cm}^{3}$ ), 5-FU monotherapy (group 4; $1.76 \pm 0.52 \mathrm{~cm}^{3}$ ), and, finally, the placebo control group (group $1 ; 3.98 \pm 0.60 \mathrm{~cm}^{3}$; Figure 4A). One-way ANOVA revealed a statistically significant difference across all groups $\left(F_{3,16}=40.09, P<0.001\right)$ as well as between groups (Supplemental Table 3). These results show that PRL3-zumab has more therapeutic efficacy when used without the chemotherapeutic drug, 5-FU.

The host immune system plays an important role in the efficacy of PRL-3 antibody therapy (5). In light of the known side effects of 5-FU treatment in causing a reduction in the numbers of white blood cells (wbc) (38), we investigated whether the reduction in therapeutic efficacy observed might be due to this phenomenon. In whole-blood smears, we found a 5 -fold reduction in peripheral wbc counts after 5-FU treatment 
A

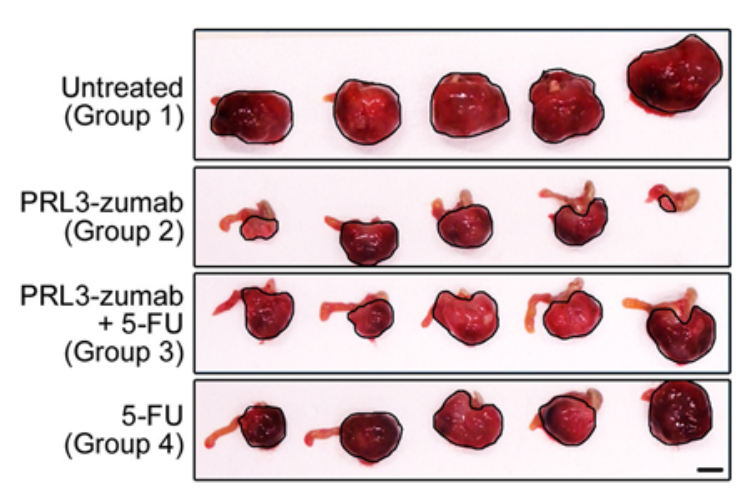

Tumor vol. $\left(\mathrm{cm}^{3}\right)$

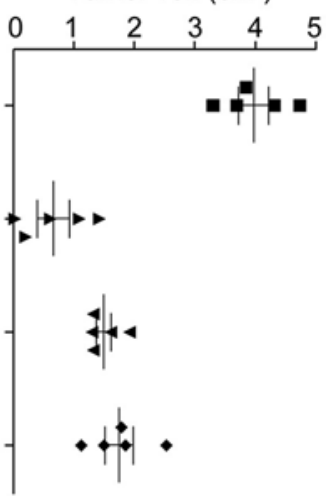

B

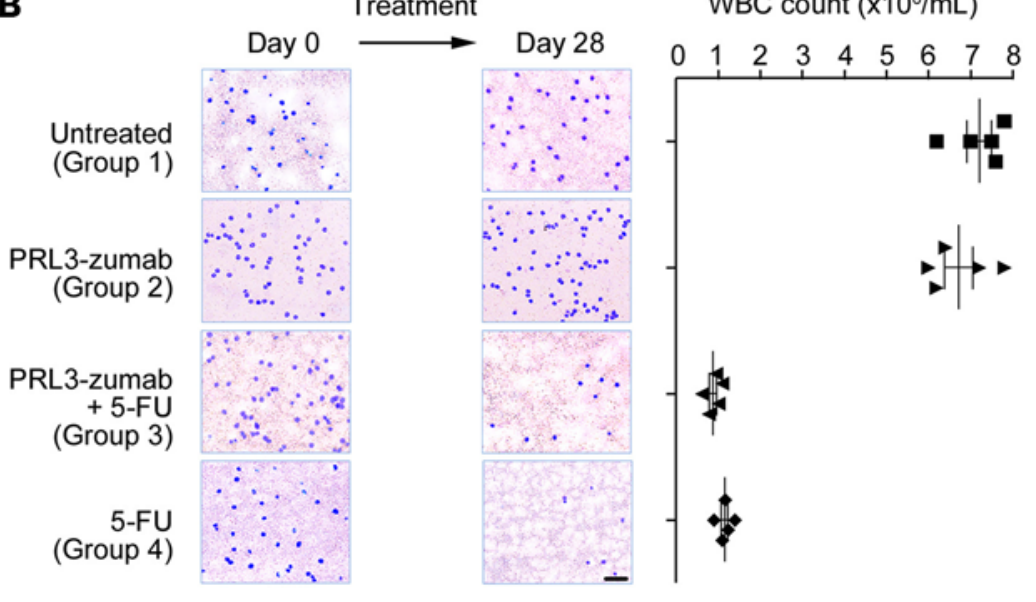

Table 2. Hematological profiles of mouse groups at the end of the various treatment regimens (day 28)

\begin{tabular}{lccccc}
\hline \multicolumn{1}{c}{ Hematology } & $\begin{array}{c}\text { Reference } \\
\text { range }\end{array}$ & Unit & PRL3-zumab & $\begin{array}{c}\text { PRL3-zumab } \\
+ \text { 5-FU }\end{array}$ & $\begin{array}{c}\text { 5-FU } \\
\text { alone }\end{array}$ \\
wbc count & $3.48-14.03$ & $\times 10^{9} / 1$ & 4.83 & $1.10^{\mathrm{A}}$ & $0.60^{\mathrm{A}}$ \\
Neutrophils & $0.58-3.83$ & $\times 10^{9} / \mathrm{l}$ & 1.65 & $0.04^{\mathrm{A}}$ & $0.03^{\mathrm{A}}$ \\
Lymphocytes & $2.22-9.83$ & $\times 10^{9} / \mathrm{l}$ & 3.03 & $0.98^{\mathrm{A}}$ & $0.54^{\mathrm{A}}$ \\
Monocytes & $0.21-1.25$ & $\times 10^{9} / \mathrm{l}$ & 0.16 & $0.03^{\mathrm{A}}$ & $0.03^{\mathrm{A}}$ \\
Eosinophils & $0.01-0.49$ & $\times 10^{9} / \mathrm{l}$ & 0.00 & 0.02 & $0.00^{\mathrm{A}}$ \\
Basophils & $0.00-0.18$ & $\times 10^{9} / 1$ & 0.01 & 0.04 & 0.01 \\
RBC count & $6.93-12.24$ & $\times 10^{12} / \mathrm{l}$ & 6.97 & $5.07^{\mathrm{A}}$ & $4.87^{\mathrm{A}}$ \\
Hemoglobin & $12.6-20.5$ & $\mathrm{~g} / \mathrm{dl}$ & $10.4^{\mathrm{A}}$ & $7.4^{\mathrm{A}}$ & $7.1^{\mathrm{A}}$ \\
MCV & $50.7-64.5$ & $\mathrm{fl}$ & 58.3 & $49.3^{\mathrm{A}}$ & $47.7^{\mathrm{A}}$ \\
MCH & $13.2-17.6$ & $\mathrm{pg}$ & 15.0 & 14.7 & 14.7 \\
MCHC & $23.3-32.7$ & $\mathrm{~g} / \mathrm{dl}$ & 26.0 & 29.3 & 21.7 \\
Platelet & $420-1,698$ & $\times 10^{9} / \mathrm{l}$ & 1,016 & $202^{\mathrm{A}}$ & $198^{\mathrm{A}}$
\end{tabular}

${ }^{A}$ Outliers from the normal reference range for BALB/c nude mice. Data are presented as mean values; $n=3$ per group.
Figure 4. PRL3-zumab is more effective as a monotherapy rather than when coadministered with 5-fluorouracil or 5-fluorouracil alone. Four treatment groups were used to treat PRL-3+ SNU-484 orthotopic tumors: placebo (group 1), PRL3-zumab monotherapy (group 2), PRL3-zumab and 5-fluorouracil (5-FU) combination therapy (group 3), or 5-FU alone (group 4). (A) Excised stomachs from each group of mice at day 28, with orthotopic tumor areas framed with black lines, and mean gastric tumor volumes in each group at day 28. Scale bar: $10 \mathrm{~mm} . n=5$ per group; $P<0.001$, 1-way ANOVA; data represent mean \pm SEM. (B) Representative images of Giemsa-stained blood smears from each group before the start of therapy (day 0 ) and at the end of the experiment (day 28) and mean wbc count from blood smears from each mouse at day 28. wbc are stained blue. Scale bar: $40 \mu \mathrm{m} . n=5$ per group; $P<$ 0.001 , 1-way ANOVA; data represent mean $\pm \mathrm{SEM}$.

(groups 3 and 4) compared with control (group 1) or PRL3-zumab monotherapy (group 2; Figure 4B). Although 1-way ANOVA analysis revealed a statistically significant difference across all groups $\left(F_{3,16}=224.26, P<0.001\right)$, no significant difference in wbc counts was found between control and PRL3-zumab monotherapy groups (Supplemental Table 4; $P=0.438$ ), suggesting that wbc counts were unaffected by PRL3-zumab treatment. To validate these results, we performed full blood counts at the end of the experiment (day 28) to analyze the hematological effects of the different treatment regimens. Whereas mice receiving PRL3-zumab had a general hematological profile within the normal reference range for the BALB/c nude strain (39), those receiving 5-FU in combination with PRL3-zumab, or 5-FU alone, displayed pronounced leucopenia, with reduced neutrophil, lymphocyte, and monocyte counts as well as marked reductions in rbc and platelets (Table 2). Along with this hematological defect, during the course of the experiment, we also observed a reduction in overall animal activity in 5-FU-treated mice. Taken together, our results suggest that the reduction in immune function as a result of 5-FU treatment may account for the reduced efficacy of PRL3-zumab when used in combination with 5-FU.

PRL3-zumab therapy suppresses recurrence of postoperative PRL- $3^{+}$tumors. Although surgery is the cornerstone in the treatment of GC, nearly $80 \%$ of patients die within a short period of time after surgery, largely due to locoregional recurrence and/or, to a lower extent, distant metastasis (40). In light of PRL3-zumab's ability to suppress PRL-3 ${ }^{+}$GC growth in vivo, we investigated whether PRL3-zum$\mathrm{ab}$ also had efficacy as a postoperative adjuvant treatment to suppress tumor recurrence. Using PRL-3 ${ }^{+}$SNU-484 GC cells, we first established 
A

INOCULATION
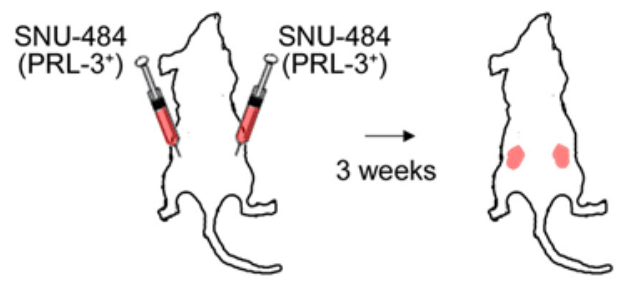

$\downarrow$

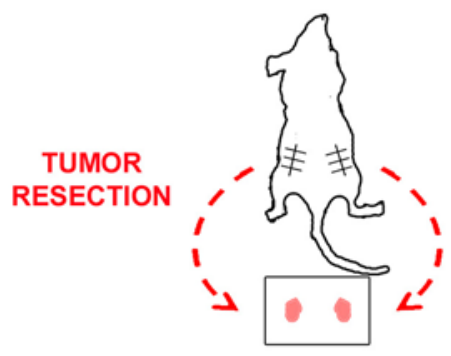

Placebo or PRL3-zumab $\downarrow \begin{aligned} & \text { Up to } \\ & 10 \text { weeks }\end{aligned}$

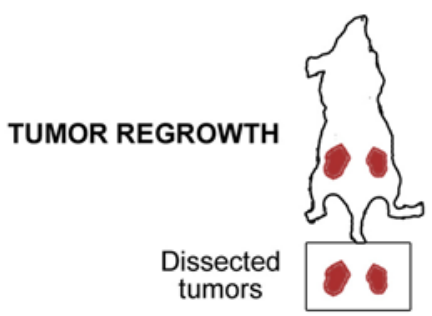

B

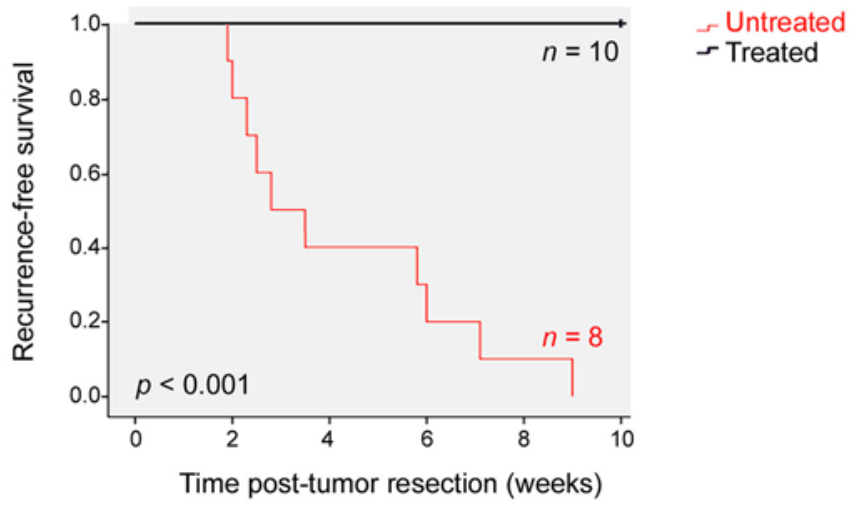

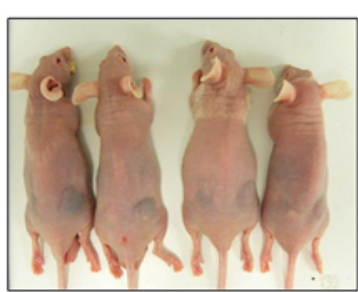
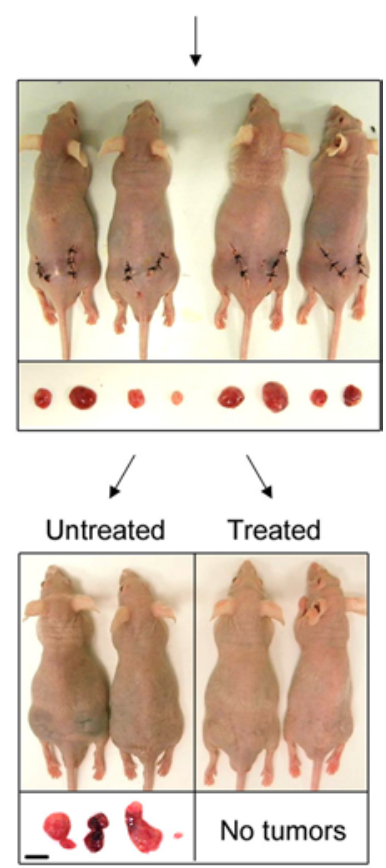

xenografts in both flanks of nude mice over the course of 3 weeks (Figure 5A, top image). The resulting tumors were then completely removed via careful surgical resection (Figure 5A, middle image), and mice were injected biweekly, for 5 weeks, with either placebo (untreated) or PRL3-zumab (treated). Tumor recurrence, defined herein as regrowth of tumors with diameter greater than or equal to $0.5 \mathrm{~cm}$ at each resection site, was monitored for up to 10 weeks after resection. Whereas all untreated mice had recurrent tumors at resection sites, none were observed in treated mice over the 10-week study duration (Figure 5A, bottom image). Accordingly, treated mice had a significantly longer recurrence-free survival compared with untreated mice (Figure 5B; Kaplan-Meier survival analysis, $P<0.001$ ). Collectively, these results suggest that PRL3-zum$a b$ has efficacy in suppressing postoperative local tumor recurrence.

PRL3-zumab suppresses growth and regional spread of xenograft PRL-3 $3^{+}$tumors in a stomach colonization model. Metastasis to the stomach is a rare condition (41-43), which is almost invariably associated with poor prognosis $(44,45)$. To indi-

rectly address whether PRL3-zumab could block stomach colonization by disseminated, non-GC cells, we developed a model using PRL-3 ${ }^{+}$HCT116-luc2 colorectal cancer cells (29) surgically implanted within the subserosa layer of mouse stomachs. We chose HCT116-luc2 cells for this model because (a) colorectal cancer metastasis to stomach is clinically relevant $(42,43,46)$, (b) HCT116-luc2 cells robustly and rapidly form tumors within the gastric niche, and (c) HCT116-luc2 cells constitutively express firefly luciferase, allowing noninvasive monitoring of tumor growth using an In Vivo Imaging System (IVIS). In two biological replicates, whereas PRL- $3^{+}$HCT116-luc2 colorectal cancer cells rapidly established large tumors within the abdominal region of untreated mice, mice treated with PRL3-zumab had suppressed PRL-3 $3^{+}$tumor growth (Supplemental Figure 5A). Two-way ANOVA analysis of the change in global tumor luminescence intensities over 3 weeks revealed a statistically significant difference between untreated and treated groups (Supplemental Figure 5B; $F_{2,18}=16.731, P<0.001$ ). Heavier HCT116-luc2 tumor burden was confirmed 
A

INTRACELLULAR PROTEINS

(after $48 \mathrm{~h}$ culture in serum-free media)

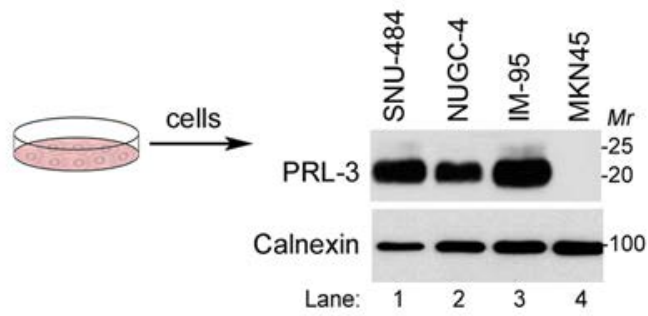

EXTRACELLULAR PROTEINS

(after $48 \mathrm{~h}$ culture in serum-free media)

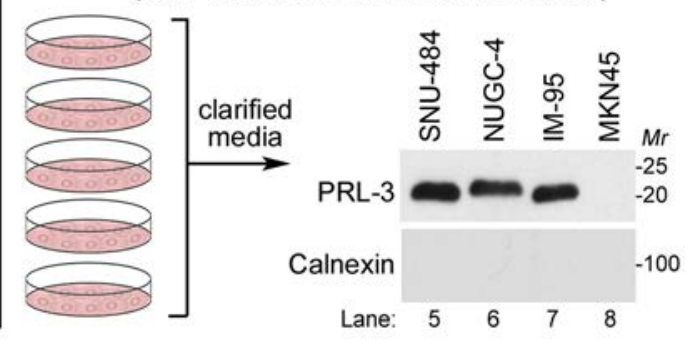

B

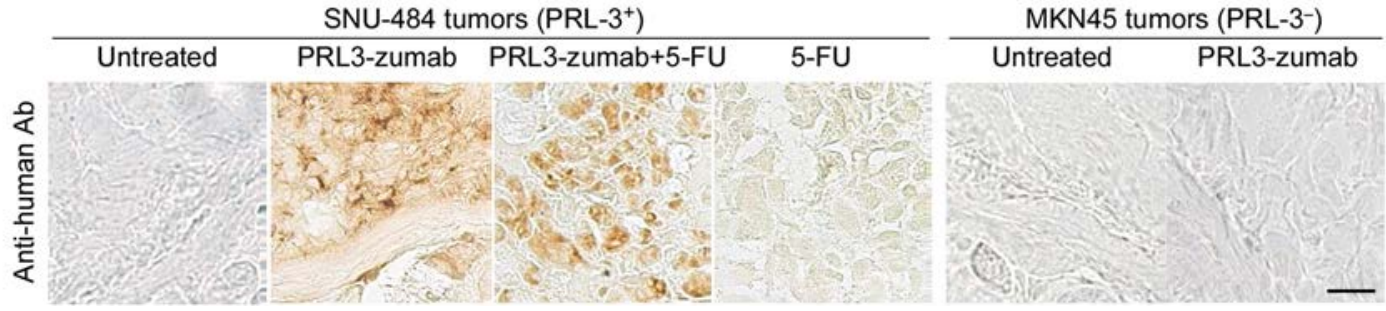

Figure 6. PRL-3 oncoprotein may be secreted out of cancer cells and act as bait for PRL3-zumab. (A) Analysis of PRL-3 protein expression in intracellular protein pools (cell lysate) and extracellular protein pools (concentrated conditioned media) after culturing gastric cancer (GC) cells in serum-free media for 48 hours. For extracellular protein analysis, conditioned media $(50 \mathrm{ml})$ from 5 dishes of GC cells were first cleared of dead cells and cellular debris, followed by centrifugal concentration (final volume $\sim 0.2 \mathrm{ml}$ ). Mr, relative molecular mass (kDa). (B) Orthotopic SNU-484 and MKN45 tumor tissue cryosections from mice subject to various treatments were analyzed by immunohistochemistry for PRL3-zumab using anti-human IgC antibodies. Scale bar: $20 \mu \mathrm{m}$.

in stomachs of untreated mice compared with treated mice (Supplemental Figure 5C), with significantly smaller gastric tumors in the latter (Supplemental Figure 5D; $P<0.001$ ). Furthermore, the extensive metastatic tumors attached to abdominal walls seen in untreated mice were also significantly reduced in treated mice (Supplemental Figure 5, E and F; $P=0.0003$ ). These results demonstrate that PRL3-zumab can also reduce growth and regional spreading of secondary tumors in and around the gastric niche.

Possible mechanism of action for PRL3-zumab suppression of PRL-3+ tumors. Studies on autoimmune pathologies have shown that autoantibodies can bind specific intracellular antigens and accumulate within the cytoplasm and nuclear compartments of antigen-expressing cells (47). Likewise, we have observed that antiPRL-3 antibodies can be internalized by PRL- $3^{+}$tumor cells in vitro (4). However, the mode of antibody uptake remains poorly defined. Here, we uncover two findings by which intracellular PRL-3 antigens might engage antibodies for specific binding and tumor suppression. (a) Intracellular PRL-3 oncoprotein can be secreted out. In tumor cells, several classically "intracellular" proteins have been reported to be externalized via secretion and/or cell surface relocalization, thereby making them accessible to therapeutic intervention using antibodies $(48,49)$. We investigated whether PRL-3 might likewise be externalized as a target antigen for PRL3-zumab binding by comparing PRL-3 intracellular and extracellular PRL-3 expression in three PRL-3+ ${ }^{+}$cell lines, SNU-484, NUGC-4, and IM-95, and one PRL-3- cell line, MKN45. PRL-3 expression was compared with the nonsecreted, ER-anchored protein, calnexin, as a control. PRL-3 protein was detected both in intracellular protein fractions (cell lysates) of PRL-3 $3^{+}$GC cells (Figure 6A, lanes 1-3) as well as in extracellular protein pools (concentrated conditioned media) of PRL-3 $3^{+} \mathrm{GC}$ cells (Figure 6A, lanes 5-7) but not PRL-3- GC cell lines (Figure 6A, lanes 4 and 8). In contrast, calnexin was exclusively present in intracellular pools of both PRL-3+ and PRL-3- GC cells (Figure 6A, lanes 1-4) but not in extracellular pools (Figure 6A, lanes 5-8). This observation ruled out nonspecific contamination by dead cells or cellular debris and characterizes PRL-3 as a novel secreted protein. (b) Externalized PRL-3 may serve as bait for PRL3-zumab binding. We next investigated the tumor sections from treated orthotopic GC mice and analyzed the distribution of PRL3-zumab within the tumor niche. As a control, no signals were seen in untreated mice (Figure 6B, leftmost panels). Following treatment, PRL3-zumab was enriched within PRL-3 ${ }^{+}$ SNU-484 tumor microenvironments, regardless of 5-FU coadministration (Figure 6B). In contrast, no enrichment was observed in PRL-3- MKN45 tumor microenvironments (Figure 6B). These results indicated a specific accumulation of PRL3-zumab in the microenvironment of PRL- $3^{+}$tumors but not PRL-3- tumors. 


\section{Discussion}

This study characterizes PRL-3 as a tumor-specific oncotarget, establishes orthotopic gastric models for proof-of-concept preclinical studies, and establishes PRL3-zumab as a clinically translatable drug candidate against PRL- $3^{+}$tumors. We demonstrated the marked therapeutic efficacy of PRL3-zumab directed against intracellular PRL-3-expressing primary gastric tumors and secondary colorectal tumors within the stomach niche as well as its value as an adjuvant in postoperative therapy to prevent tumor recurrence. Conceptually, this study further demonstrates the previously unrecognized potential of tumor-specific intracellular oncoproteins as viable molecular targets for specific antibody cancer therapy.

To create a clinically relevant orthotopic GC model using human GC cell lines, we employed immunodeficient nude mice instead of severely immunocompromised mice strains, such as NOD/SCID, BAL$\mathrm{B} / \mathrm{c}-\mathrm{RAG} 2^{\text {null }}$, or their derivatives (50). These latter strains have little or no endogenous immune system intact, creating a gap in translating research findings to immunocompetent human patients. The use of a more clinically relevant mouse model also overcomes the limitations of in vitro drug screening in cell culture systems, which are unable to recapitulate the complex interactions within the body and are poorly predictive of in vivo toxicity (51). Our finding of secreted PRL-3 oncoprotein sheds light onto the recognition mechanism for antibody binding and targeting to PRL- $3^{+}$tumors. The specific externalization of classically "intracellular" proteins in cancer cells and their viability as therapeutic antibody targets have been well studied $(48,49,52)$. Indeed, marked amounts of intracellular material have long been reported to be spontaneously released from malignant cells into tumor microenvironments and extracellular fluids $(53,54)$. It is reasonable to anticipate that in vivo greater secretion of intracellular oncoproteins occurs due to tumor tissues consistently being under immune surveillance and attack, thereby establishing an inflammatory state that results in apoptosis and/or necrosis of tumor cells (55) and further nonspecific leakage of intracellular content. Recognition of antibodies by immune effector cells occurs via immunoglobulin receptors (FcRs), which bind the Fc portion of antibodies, resulting in recruitment and activation of effector cells (56), such as $\mathrm{B}$ and NK cells, two FcR-bearing immune cell types suggested to be involved in intracellular antibody therapy (7). Under such conditions, externalized "intracellular" tumor antigens could be recognized by antibodies in vivo to (a) induce antibody-dependent cellular cytotoxicity by recruitment of NK cells, (b) form immune complexes (for secreted antigens) that can then be processed by regional dendritic cells for presentation and activation of NK cells, or (c) target cells for antibody-dependent cellular phagocytosis by macrophages, which has lately been characterized as a major mechanism of action behind many antibodies approved to treat cancer (57). Supporting this hypothesis, anti-PRL-3 antibodies have been shown to lack anticancer efficacy in immunocompromised SCID mice (5), or when directly added to PRL-3 $3^{+}$cancer cells in vitro (29), indicating the importance of the interaction of the therapeutic agent with immune effectors for successful treatment. Interestingly, PRL-3 was recently shown to promote secretion of ULBP2, an NKG2D ligand, resulting in reduced tumor recognition and cytolysis by NK cells (58). Nonetheless, the effective tumor suppression seen in PRL3-zumab-treated mice suggests that PRL3-zumab allows the host to overcome this immune evasion mechanism, resulting in more efficient targeting of PRL- $3^{+}$tumors.

In our study, PRL-3 oncoprotein was overexpressed in greater than $80 \%$ of the GC samples examined. With such a high PRL-3 tumor positivity, the development of PRL3-zumab-targeted therapy against tumor-specific PRL-3 is an exciting step towards personalized medicine. By maximizing therapeutic benefits and minimizing off-target side effects - PRL-3 is not expressed at detectable levels in most normal adult tissues - PRL3-zum$\mathrm{ab}$ justifies clinical validation and development as a precision anticancer drug. Although our results here focus on GC, $P R L-3$ has also been extensively linked to multiple cancer types of tumor metastasis and poor prognosis, with higher PRL-3 expression associated with shorter overall survival (18). Based on the principle that PRL3-zumab exerts its effects only upon recognizing PRL-3 antigen, it is envisaged to have efficacy in targeting most, if not all, PRL- $3^{+}$cancers, opening a new therapeutic avenue in general cancer therapy.

In summary, our findings here and elsewhere $(4,5,29)$ challenge the dogma that intracellular oncoantigens are inaccessible to therapeutic antibodies for anticancer effects. We suggest that other intracellular tumor-specific antigens could likewise have tremendous potential for targeted immunotherapy (6), prompting their reevaluation as tractable molecular targets in future clinical trials.

\section{Methods}

Preparation of tissue and cell lysates. Multiple normal mouse organs were harvested from FVB/wild-type mice, whereas breast and metastatic lung tumors were dissected from the isogenic FVB/MMTV-PyMT 
mice strain - a well-established spontaneous model of metastatic breast cancer driven by transgenic overexpression of mammary-specific polyoma virus middle $\mathrm{T}$ oncogene (30) - obtained from the Biological Resource Centre (A*STAR). For tissues, excised samples $\left(5 \mathrm{~mm}^{3}\right)$ were suspended in RIPA lysis buffer (Sigma-Aldrich) containing a protease-phosphatase inhibitor cocktail (Pierce) and disrupted completely with a tissue homogenizer (Polytron). Lysates were clarified by centrifugation at $13,000 \mathrm{~g}$ for 40 minutes at $4^{\circ} \mathrm{C}$. For cell lines, $5 \times 10^{6}$ cells were lysed in RIPA lysis buffer containing a protease-phosphatase inhibitor and clarified as described above. Protein concentrations were estimated using a bicinchoninic assay kit (Pierce). After addition of $2 \times$ Laemmli buffer, samples were boiled and used immediately for Western blotting or stored at $-20^{\circ} \mathrm{C}$ until use.

Western blotting. Tissue lysates ( $40 \mu \mathrm{g})$ or cell lysates $(200 \mu \mathrm{g})$ were resolved in $12 \%$ SDS-polyacrylamide gels and transferred to nitrocellulose membranes before blocking and probing with the indicated primary antibodies at a 1:1,000 dilution overnight at $4^{\circ} \mathrm{C}$. After thorough washing with TBS-T buffer (20 mM Tris $\mathrm{pH} 7.6,140 \mathrm{mM} \mathrm{NaCl}, 0.2 \%$ Tween-20), the membrane was incubated with the respective HRP-conjugated secondary antibodies at a 1:5,000 dilution for 1 hour, washed with TBS-T buffer, and visualized using a chemiluminescent substrate (Pierce). Band intensities were calculated using ImageJ software (NIH).

Cell culture. The 22 human GC cell lines studied were obtained from the following sources: MKN7, MKN74, NUGC-3, and OCUM-1 (Health Science Research Resources Bank); YCC-1, YCC-3, YCC-7, and YCC-17 cells (Yonsei Cancer Centre); AGS, CRL-5822, KATO-III, SNU-1, and SNU-5 (ATCC); HGC27, NUGC-4, and OE19 (Sigma-Aldrich); MKN28 and MKN45 (RIKEN BioResource Center); IM-95 and SCH (Japanese Collection of Research Bioresources Cell Bank); and SNU-484 and SNU-719 (Korean Cell Line Bank). CHO cells were purchased from ATCC. The generation of CHO cells stably expressing GFPtagged PRL-1, PRL-2, or PRL-3 fusion proteins has been previously described (4). CHO and MKN45 cells stably expressing GFP-tagged isoforms of human PRL-1, PRL-2, or PRL-3 were similarly established by transfection with plasmids generated by subcloning the respective human ORFs from previously generated pGEX-KG-PRL-1, pGEX-KG-PRL-2, or pGEX-KG-PRL-3 vectors (59) into pEGFP-C1 vectors (Clontech), followed by selection in $1 \mathrm{mg} / \mathrm{ml} \mathrm{G} 418$ (Sigma-Aldrich) for 2 weeks. Luciferase-expressing HCT116luc2 human adenocarcinoma cells (Caliper Life Sciences) were established by stably transducing lentivirus containing the luciferase 2 gene under the control of human ubiquitin C promotor (pGL4 luc2) into parental HCT116 cells (ATCC). Cell lines were cultured in RMPI-1640 medium (Gibco) supplemented with $10 \%$ heat-inactivated fetal bovine serum (Hyclone) and 1\% penicillin-streptomycin (Life Technologies) and maintained in a $37^{\circ} \mathrm{C}$ incubator supplemented with $5 \% \mathrm{CO}_{2}$.

Generation of PRL3-zumab. The PRL3-zumab construct was engineered from the original framework of the previously characterized murine anti-PRL-3 antibody clone 318 (59) independently by two CROs using a proprietary modification of the method described by Queen et al. (60). Briefly, the complementarity determining regions (CDR) of the heavy (IgG1) and light $(\kappa)$ chains of the mouse antibody were grafted onto "acceptor" human sequence frameworks, where the framework is defined as the segment of the variable regions excluding the CDRs. The choice of human acceptor frameworks was made by aligning the mouse framework sequences against a database of human framework sequences to find the closest human homolog for each chain (typically $65 \%-70 \%$ sequence identity). In addition to grafting the CDRs from the mouse sequence, about 3 amino acid positions from the mouse sequence (in addition to the CDRs) were also grafted into the human acceptor sequence. This preserved the original murine anti-PRL3 antibody's CDR, which specifically recognizes an epitope within a C-terminal region conserved between both mouse and human PRL-3, but not PRL-1 or PRL-2. PRL3-zumab was subsequently purified from the culture media of stably transfected $\mathrm{CHO}$ master cell lines. Binding affinity analysis, conducted by Sapidyne Instruments using a kinetic exclusion assay (61), characterized purified PRL3-zumab to have a $K_{D}$ of $6.29 \mathrm{pM}$ to purified human PRL-3, with an on rate $\left(K_{\text {on }}\right)$ and off rate $\left(K_{\text {off }}\right)$ of approximately $1 \times 10^{7}$ $\mathrm{M}^{-1} \mathrm{~s}^{-1}$ and $7 \times 10^{-5} \mathrm{~s}^{-1}$, respectively.

Analysis of PRL-3 mRNA expression. We analyzed the publically available GC microarray data set (GSE15459) from the Gene Expression Omnibus database, consisting of 200 primary GC specimens profiled on Affymetrix Human Genome U133 Plus 2.0 Genechip arrays. Data preprocessing was carried out using the "affyPLM" R package (v2.15). Outliers were excluded, giving a total of 185 tumor samples available for downstream analyses (SGset1; patient characteristics are provided in Supplemental Table 1). Survival analyses, with overall survival as the outcome metric, were performed to compare tumors $(n=183$; 2 samples missing survival data), with "low," "medium," and "high" expression of the respective genes, 
i.e., "low" and "high" expression groups correspond to samples with lower than the 33.3 percentile expression levels and greater than the 66.7 percentile expression levels, respectively, while the middle percentile was classified as "medium."

Preparation of recombinant GST-tagged proteins. The preparation of recombinant GST-PRL-1, GSTPRL-2, and GST-PRL-3 fusion proteins has been described previously (59).

ELISA. ELISA was performed as described previously (59). Briefly, 96-well plates coated overnight with GST-PRL-1 (20 ng), GST-PRL-2 (20 ng), or GST-PRL-3 (1 ng) were blocked with 3\% bovine serum albumin in PBS- $0.05 \%$ Tween-20 prior to incubation with 200 ng PRL3-zumab for 2 hours at $37^{\circ} \mathrm{C}$. After extensive washing, HRP-conjugated anti-mouse antibody (Pierce) was added for 1 hour at $37^{\circ} \mathrm{C}$. Colorimetric development was performed using a Turbo-TMB substrate (Pierce) and stopped by acidification with $2 \mathrm{M} \mathrm{H}_{2} \mathrm{SO}_{4}$. Absorbance was measured at $450 \mathrm{~nm}$ using a plate reader (Dynatech).

Animal models. Eight-week-old male BALB/c nude mice obtained from the Biological Resource Centre (A*STAR) were used for all animal models in this study. Mice were anesthetized with $2.5 \%$ avertin (100 $\mu 1 / 10$ g body weight).

Orthotopic GC model. Abdomens of anesthetized mice were opened in layers by a 1-cm midline incision starting from $0.5 \mathrm{~cm}$ below the xiphoid sternum. The stomach was taken out through the abdominal incision by surgical forceps, and equal numbers of cancer cells from a master cell pool were injected into the subserosa layer. The cell numbers required to induce orthotropic gastric tumors for each cell line and the duration of experiments were confirmed after preliminary experiments: $3 \times 10^{6}$ cells for SNU-484 tumors and $5 \times$ $10^{6}$ cells for IM-95, NUGC-4, MKN45, and MKN45-PRL-3 tumors. Subsequently, the stomach was placed back and the abdomen was sutured back in layers. Because of the different growth rates of the individual tumors, the duration of experiments were as follows: 4 weeks for SNU-484, NUGC-4, and MKN45-PRL-3 tumors, 8 weeks for MKN45 tumors, and 12 weeks for IM-95 tumors. The treatment regime commenced on day 2 after inoculation of cancer cells in the gastric subserosa layer. For tumor growth/volume experiments, treated mice were administered i.v. with $100 \mu \mathrm{g}$ of PRL3-zumab or, where indicated, human IgG isotype control (catalog BE0092; Bio X Cell) diluted in $100 \mu 1$ PBS twice a week, for a total of 8 times (SNU-484, NUGC-4, MKN45-PRL-3 tumors) or 10 times (IM-95, MKN45 tumors). Where indicated, 5-FU (Hospira) was administered i.v. via tail vein either alone or together with PRL3-zumab at a dose of $30 \mathrm{mg} / \mathrm{kg}$ biweekly for a total of 8 times. Tumor volumes were calculated using the formula volume $=0.4 \times$ tumor length $\times$ tumor width $\times$ tumor width (62). For survival studies, treated mice were administered i.v. with $100 \mu \mathrm{g}$ of PRL3-zumab diluted in $100 \mu \mathrm{PBS}$ twice a week, for a total of 10 times. Untreated mice were administered i.v. with an equivalent volume of placebo (buffer alone) as a control. When mice displayed reduced physical activity and appeared ill, they were euthanized.

Xenograft tumor recurrence model. $3 \times 10^{6}$ SNU-484 cells in $150 \mu \mathrm{l}$ of PBS were injected into both flanks of anesthetized mice. After 3 weeks, resultant tumors (0.5- to 1-cm diameter) were surgically removed under anesthesia, and mice were divided into treated or untreated groups to receive biweekly doses of either PRL3-zumab or placebo, respectively. Tumor recurrence, defined as the regrowth of tumor with diameter greater than or equal to $1 \mathrm{~cm}$ at resection sites, was then monitored weekly in both groups up till 10 weeks after resection. Tumor size was measured using calipers. Recurrence-free survival was analyzed by the Kaplan-Meier method.

Stomach xenograft colonization model. $3 \times 10^{6} \mathrm{HCT} 116-1 \mathrm{uc} 2$ cells were directly implanted into the gastric serosa of anesthetized mice, as described above. Mice were divided into treated or untreated groups, and tumor growth at weeks 1, 2, and 3 after implantation was monitored by IVIS imaging under 2\% isoflurane anesthesia 15 minutes after intraperitoneal injection of $150 \mathrm{mg} / \mathrm{kg}$ luciferin (Caliper Life Sciences).

Analysis of mouse blood samples. After smearing a thin layer from a drop of fresh mouse blood on a glass slide, slides were baked at $37^{\circ} \mathrm{C}$ for 1 hour before flooding with modified Wright Giemsa stain (SigmaAldrich) for 1 minute followed by washing with deionized water for 3 minutes. After drying, the stained slides were observed under a light microscope (Olympus), with wbc stained blue. Estimation of total wbc counts was performed by taking the average of wbc from at least 10 visual fields in each slide, and calculation was performed using the equation $\mathrm{wbc} / \mu \mathrm{l}=$ (total number of counted wbc/number of fields) $\times 2,000$. Full hematological profiling of mice blood samples was conducted by Quest Laboratories.

Antibodies. Calnexin (catalog 2679) antibody was purchased from Cell Signaling. CD63 (catalog sc-15363) antibody was purchased from Santa Cruz Biotechnology. GAPDH (clone MAB374) antibody was purchased from Millipore. HSP60 (catalog 611562) and HSP70 (catalog 610607) antibodies 
were purchased from BD Pharmingen. HRP-conjugated anti-mouse (catalog 715-035-150), anti-rabbit (catalog 711-035-152), and anti-human (catalog 709-035-149) secondary antibodies were purchased from Jackson ImmunoResearch Inc.

Conditioned media analysis. Exponentially growing cells at $70 \%$ to $80 \%$ confluence in 5 T-75 culture flasks (Corning) were washed twice with serum-free RPMI before incubation with serum-free RPMI for 48 hours. Conditioned media were centrifuged twice at 1,800 $\mathrm{g}$ for 10 minutes to remove floating cells and cellular debris. Clarified supernatants $(50 \mathrm{ml}$ ) were subsequently concentrated $\sim 250$-fold (final volume $\sim 0.2$ $\mathrm{ml}$ ) using $3 \mathrm{~K}$ MWCO spin concentrators (Millipore), quantitated, and analyzed by Western blotting.

Immunofluorescence imaging. Cells were seeded directly onto glass coverslips and grown for 48 hours. After washing twice with PBSCM (PBS pH 7.0, $1 \mathrm{mM} \mathrm{MgCl}_{2}, 1 \mathrm{mM} \mathrm{CaCl}$ ), cells were fixed in $3 \%$ paraformaldehyde (Sigma-Aldrich) for 20 minutes at room temperature, washed, permeabilized for 15 minutes with PBS-0.1\% saponin (Sigma-Aldrich), and blocked in PBS-FDB (PBS pH 7.0, 2\% BSA, 5\% goat serum, $5 \%$ fetal bovine serum) for 1 hour at room temperature. Slides were subsequently incubated with PRL3-zumab at a 1:200 dilution at room temperature for 4 hours, washed, and incubated for 2 hours with the AlexaFluor546-conjugated goat anti-human secondary antibodies (catalog A-21089; ThermoFisher Scientific). Washed slides were mounted with a DAPI-containing anti-fade mounting reagent (Vector Laboratories) and sealed using nail polish. Confocal imaging was performed with an LSM 510 confocal microscope (Zeiss AG).

Immunohistochemistry. To analyze PRL-3 expression in normal human tissues, we used a commercial tissue microarray (catalog NC00-02-009; Cybrdi) containing 100, 4- $\mu$ m-thick sections from different individuals verified for normal histology across 33 different tissue types. For tumor specimens, $10-\mu \mathrm{m}$-thick cryosection slides were fixed with $4 \%$ formalin for 20 minutes and incubated with $1 \% \mathrm{H}_{2} \mathrm{O}_{2}$-PBS in the dark for 5 minutes. Washed slides were then blocked in PBS containing 10\% goat serum and 1\% BSA (Sigma-Aldrich) for 1 hour at room temperature. Subsequently, slides were washed 3 times in PBS- $0.05 \%$ Tween-20 with gentle shaking and incubated with mouse anti-PRL-3 antibody (1:100 dilution) overnight at $4^{\circ} \mathrm{C}$. After washing, HRP-labeled polymer (anti-mouse; Dako) was added for 2 hours before washing extensively and incubating with substrate-chromogen solution (Dako) for 10 to 20 minutes in the dark. Mounted slides were examined using a bright-field microscope (Olympus), and representative images were captured. For direct detection of PRL3-zumab in tissues, slides were fixed and blocked as described above, incubated with HRP-labeled polymer (anti-human; Dako) without primary antibodies, and subsequently processed as described above.

Statistics. For human studies, the log-rank test was used to assess the significance of the Kaplan-Meier analysis of overall GC patient survival, based on PRL-3 mRNA expression grouping. Univariate and multivariate analyses were performed using Cox proportional hazards regression. For mouse studies, the log-rank test was used to assess significant differences in the Kaplan-Meier analysis of overall survival between untreated and treated mouse groups. Tumor volumes and stomach IVIS luminescence intensities between untreated and treated mice were analyzed using 2-tailed Student's $t$ tests. ELISA measurements, tumor volumes, wbc counts, and fluorescence intensities of B and NK cell infiltration among multiple treatment groups were separately analyzed using 1-way ANOVA and, where indicated, post-hoc Tukey's HSD test. Global IVIS luminescence intensities between treatment groups over time were analyzed by 2-way ANOVA. SPSS v19.0 (IBM) or Prism v4.0 (Graphpad Software) were used for statistical calculations. In all instances, $P$ values less than 0.05 were considered significant.

Study approval. Mouse studies were approved by the Biological Resource Centre (A*STAR) Institutional Animal Care and Use Committee and performed in accordance with their approved guidelines and regulations. Fresh-frozen primary human gastric tumors and corresponding normal tissue samples were acquired, with patient consent, from the National University Hospital-National University of Singapore Tissue Repository. The collection and use of human tissue samples were approved by the IRB of the National University of Singapore and National Healthcare Group, Singapore.

\section{Author contributions}

MT, AQOA, and QZ designed the experiments and prepared the manuscript. MT, AG, YBL, KM, SJL, LW, and JL performed the experiments. WPY, KK, JPT, BCG, PT, RS, EC, SYR, HCC, SN, HFY, SDZ, YKG, and JS provided materials and analyzed the data. MT, CWH, AQOA, and QZ analyzed data and proofread and finalized the manuscript. All authors approved the manuscript. 


\section{Acknowledgments}

This work was supported by research grants from A*STAR. We are especially grateful to Bert Vogelstein, Soldano Ferrone, and Michel Tremblay for their encouragement and continuous support for our unconventional antibody therapies. We also thank Eng Chon Boon and Rajeev Singh from the National University Hospital-National University of Singapore Tissue Repository for their help in providing clinical samples.

Address correspondence to: Qi Zeng, Institute of Molecular and Cell Biology, A*STAR, 61 Biopolis Drive, Proteos, 138673, Singapore. Phone: 65.65869664; E-mail: mcbzengq@imcb.a-star.edu.sg.

1. Weiner LM, Murray JC, Shuptrine CW. Antibody-based immunotherapy of cancer. Cell. 2012; 148(6):1081-1084.

2. Rubio-Perez C, et al. In silico prescription of anticancer drugs to cohorts of 28 tumor types reveals targeting opportunities. Cancer Cell. 2015;27(3):382-396.

3. Hoelder S, Clarke PA, Workman P. Discovery of small molecule cancer drugs: successes, challenges and opportunities. Mol Oncol. 2012;6(2):155-176.

4. Guo K, Tang JP, Tan CP, Wang H, Zeng Q. Monoclonal antibodies target intracellular PRL phosphatases to inhibit cancer metastases in mice. Cancer Biol Ther. 2008;7(5):750-757.

5. Guo K, et al. Targeting intracellular oncoproteins with antibody therapy or vaccination. Sci Transl Med. 2011; 3(99):99ra85.

6. Ferrone S. Hidden immunotherapy targets challenge dogma. Sci Transl Med. 2011;3(99):99ps38.

7. Hong CW, Zeng Q. Awaiting a new era of cancer immunotherapy. Cancer Res. 2012;72(15):3715-3719.

8. Tremblay M, Hardy S. F1000Prime Recommendation of [Guo K et al., Sci Transl Med 2011, 3(99):99ra85]. F1000Prime 2011; F1000Prime.com/13294042\#eval14653184.

9. Zeng Q, Hong W, Tan YH. Mouse PRL-2 and PRL-3, two potentially prenylated protein tyrosine phosphatases homologous to PRL-1. Biochem Biophys Res Commun. 1998;244(2):421-427.

10. Zeng Q, Si X, Horstmann H, Xu Y, Hong W, Pallen CJ. Prenylation-dependent association of protein-tyrosine phosphatases PRL-1, -2, and -3 with the plasma membrane and the early endosome. J Biol Chem. 2000;275(28):21444-21452.

11. Saha S, et al. A phosphatase associated with metastasis of colorectal cancer. Science. 2001; 294(5545):1343-1346.

12. Laurent C, et al. High PTP4A3 phosphatase expression correlates with metastatic risk in uveal melanoma patients. Cancer Res. 2011;71(3):666-674.

13. Al-Aidaroos AQO, Zeng Q. PRL-3 phosphatase and cancer metastasis. J Cell Biochem. 2010; 111(5):1087-1098.

14. Wang H, Quah SY, Dong JM, Manser E, Tang JP, Zeng Q. PRL-3 down-regulates PTEN expression and signals through PI3K to promote epithelial-mesenchymal transition. Cancer Res. 2007;67(7):2922-2926.

15. Al-Aidaroos AQ, et al. Metastasis-associated PRL-3 induces EGFR activation and addiction in cancer cells. J Clin Invest. 2013;123(8):3459-3471.

16. Zimmerman MW, et al. Protein-tyrosine phosphatase 4A3 (PTP4A3) promotes vascular endothelial growth factor signaling and enables endothelial cell motility. J Biol Chem. 2014;289(9):5904-5913.

17. Walls CD, Iliuk A, Bai Y, Wang M, Tao WA, Zhang ZY. Phosphatase of regenerating liver 3 (PRL3) provokes a tyrosine phosphoproteome to drive prometastatic signal transduction. Mol Cell Proteomics. 2013;12(12):3759-3777.

18. Bessette DC, Qiu D, Pallen CJ. PRL PTPs: mediators and markers of cancer progression. Cancer Metastasis Rev. 2008;27(2):231252.

19. Ferlay J, et al. Cancer incidence and mortality worldwide: sources, methods and major patterns in GLOBOCAN 2012. Int $J$ Cancer. 2015;136(5):E359-E386.

20. Orditura M, et al. Treatment of gastric cancer. World J Gastroenterol. 2014; 20(7):1635-1649.

21. Miskad UA, Semba S, Kato H, Yokozaki H. Expression of PRL-3 phosphatase in human gastric carcinomas: close correlation with invasion and metastasis. Pathobiology. 2004;71(4):176-184.

22. Li ZR, et al. Association of tyrosine PRL-3 phosphatase protein expression with peritoneal metastasis of gastric carcinoma and prognosis. Surg Today. 2007;37(8):646-651.

23. Dai N, Lu AP, Shou CC, Li JY. Expression of phosphatase regenerating liver 3 is an independent prognostic indicator for gastric cancer. World J Gastroenterol. 2009;15(12):1499-1505.

24. Macdonald JS, et al. Chemoradiotherapy after surgery compared with surgery alone for adenocarcinoma of the stomach or gastroesophageal junction. N Engl J Med. 2001; 345(10):725-730.

25. Cunningham D, et al. Perioperative chemotherapy versus surgery alone for resectable gastroesophageal cancer. $N$ Engl $\mathrm{J} \mathrm{Med}$. 2006; 355(1):11-20.

26. Terashima M, et al. Impact of expression of human epidermal growth factor receptors EGFR and ERBB2 on survival in stage II/III gastric cancer. Clin Cancer Res. 2012;18(21):5992-6000.

27. Janjigian YY, et al. Prognosis of metastatic gastric and gastroesophageal junction cancer by HER2 status: a European and USA International collaborative analysis. Ann Oncol. 2012;23(10):2656-2662.

28. Shimoyama S. Unraveling trastuzumab and lapatinib inefficiency in gastric cancer: Future steps (Review). Mol Clin Oncol. 2014;2(2):175-181.

29. Guo K, et al. Engineering the first chimeric antibody in targeting intracellular PRL-3 oncoprotein for cancer therapy in mice. Oncotarget. 2012;3(2):158-171.

30. Guy CT, Cardiff RD, Muller WJ. Induction of mammary tumors by expression of polyomavirus middle T oncogene: a transgenic mouse model for metastatic disease. Mol Cell Biol. 1992;12(3):954-961.

31. Zimmerman MW, Homanics GE, Lazo JS. Targeted deletion of the metastasis-associated phosphatase Ptp4a3 (PRL-3) suppresses murine colon cancer. PLoS ONE. 2013;8(3):e58300. 
32. Wang H, et al. PCBP1 suppresses the translation of metastasis-associated PRL-3 phosphatase. Cancer Cell. 2010;18(1):52-62.

33. Wang Z, et al. Expression and prognostic impact of PRL-3 in lymph node metastasis of gastric cancer: its molecular mechanism was investigated using artificial microRNA interference. Int J Cancer. 2008;123(6):1439-1447.

34. Wang Z, et al. High expression of PRL-3 can promote growth of gastric cancer and exhibits a poor prognostic impact on patients. Ann Surg Oncol. 2009;16(1):208-219.

35. Zhao S, et al. Regulation of cellular metabolism by protein lysine acetylation. Science. 2010;327(5968):1000-1004

36. Fiordalisi JJ, Dewar BJ, Graves LM, Madigan JP, Cox AD. Src-mediated phosphorylation of the tyrosine phosphatase PRL-3 is required for PRL-3 promotion of Rho activation, motility and invasion. PLoS ONE. 2013;8(5):e64309.

37. Wilmanns C, Fan D, O'Brian CA, Bucana CD, Fidler IJ. Orthotopic and ectopic organ environments differentially influence the sensitivity of murine colon carcinoma cells to doxorubicin and 5-fluorouracil. Int J Cancer. 1992;52(1):98-104.

38. Rasmussen L, Arvin A. Chemotherapy-induced immunosuppression. Environ Health Perspect. 1982; 43:21-25.

39. BALB/c Nude Mouse Hematology. Charles River Laboratories International Inc. 2012. http://www.criver.com/files/pdfs/ rms/balbc-nude/rm_rm_r_balb-c_nude_mouse_clinical_pathology_data.aspx. Accessed on May 31, 2016.

40. Sano T, et al. Gastric cancer surgery: morbidity and mortality results from a prospective randomized controlled trial comparing D2 and extended para-aortic lymphadenectomy--Japan Clinical Oncology Group study 9501. J Clin Oncol. 2004; 22(14):27672773.

41. Menuck LS, Amberg JR. Metastatic disease involving the stomach. Am J Dig Dis. 1975;20(10):903-913.

42. Green LK. Hematogenous metastases to the stomach. A review of 67 cases. Cancer. 1990;65(7):1596-1600.

43. Oda Kondo H, et al. Metastatic tumors to the stomach: analysis of 54 patients diagnosed at endoscopy and 347 autopsy cases Endoscopy. 2001; 33(6):507-510.

44. Saito T, Iizuka T, Kato H, Watanabe H. Esophageal carcinoma metastatic to the stomach. A clinicopathologic study of 35 cases. Cancer. 1985;56(9):2235-2241.

45. Gallino G, et al. Surgical treatment of gastric metastases from cutaneous melanoma: experience of the National Cancer Institute of Milan. Tumori. 2001;87(4):229-231.

46. Davis G, Zollinger R. Metastatic melanoma of the stomach. Am J Surg. 1960; 99:94-96.

47. Ruiz-Argüelles A, Alarcón-Segovia D. Penetration of autoantibodies into living cells, 2000. Isr Med Assoc J. 2001;3(2):121-126.

48. Weidle UH, Maisel D, Brinkmann U, Tiefenthaler G. The translational potential for target validation and therapy using intracellular antibodies in oncology. Cancer Genomics Proteomics. 2013;10(6):239-250.

49. Weidle UH, Maisel D, Klostermann S, Schiller C, Weiss EH. Intracellular proteins displayed on the surface of tumor cells as targets for therapeutic intervention with antibody-related agents. Cancer Genomics Proteomics. 2011;8(2):49-63.

50. Ito R, Takahashi T, Katano I, Ito M. Current advances in humanized mouse models. Cell Mol Immunol. 2012;9(3):208-214.

51. McKim JM. Building a tiered approach to in vitro predictive toxicity screening: a focus on assays with in vivo relevance. Comb Chem High Throughput Screen. 2010;13(2):188-206.

52. Takechi Y, Hara I, Naftzger C, Xu Y, Houghton AN. A melanosomal membrane protein is a cell surface target for melanoma therapy. Clin Cancer Res. 1996;2(11):1837-1842.

53. LeBreton E, Mouli Y. Brache J, Mirsky A, eds. Biochemistry and Physiology of the Cancer Cell. New York, New York, USA: Academic Press, 1961.

54. Holmberg B. On the in vitro release of cytoplasmic enzymes from ascites tumor cells as compared with strain L cells. Cancer Res. 1961;21:1386-1393.

55. Rock KL, Kono H. The inflammatory response to cell death. Annu Rev Pathol. 2008;3:99-126.

56. Nimmerjahn F, Ravetch JV. Fc-receptors as regulators of immunity. Adv Immunol. 2007;96:179-204.

57. Weiskopf K, Weissman IL. Macrophages are critical effectors of antibody therapies for cancer. MAbs. 2015;7(2):303-310.

58. Leung WH, et al. PRL-3 mediates the protein maturation of ULBP2 by regulating the tyrosine phosphorylation of HSP60. $J$ Immunol. 2015;194(6):2930-2941.

59. Li J, et al. Generation of PRL-3- and PRL-1-specific monoclonal antibodies as potential diagnostic markers for cancer metastases. Clin Cancer Res. 2005;11(6):2195-2204.

60. Queen C, et al. A humanized antibody that binds to the interleukin 2 receptor. Proc Natl Acad Sci U S A. 1989; 86(24):10029-10033.

61. Drake AW, Myszka DG, Klakamp SL. Characterizing high-affinity antigen/antibody complexes by kinetic- and equilibrium-based methods. Anal Biochem. 2004;328(1):35-43.

62. Attia MA, Weiss DW. Immunology of spontaneous mammary carcinomas in mice. V. Acquired tumor resistance and enhancement in strain A mice infected with mammary tumor virus. Cancer Res. 1966; 26(8):1787-1800. 